\title{
Measuring Health Inequality with Realization of Conditional Potential Life Years (RCPLY)
}

\author{
Dennis Petrie*, a Kam Ki Tang ${ }^{\text {b }}$, D. S. Prasada Rao ${ }^{\mathrm{b}}$
}

a School of Population and Global Health, The University of Melbourne, Australia

b School of Economics, The University of Queensland, Australia

* Corresponding author. Email: kk.tang@uq.edu.au; telephone: 6173365 9796; fax: 61733657299

\section{Acknowledgements}

The authors would like to acknowledge helpful comments by Roberto Zelli on an earlier version of this paper. Kam Ki Tang would also like to acknowledge the support by a grant from Australian Research Council (DP0878752).

Measuring Health Inequality with

Realization of Conditional Potential Life Years (RCPLY)

\begin{abstract}
Health inequalities that are avoidable and unfair are considered more relevant for policy intervention. Recent work has improved on using life years (LY) to measure longevity inequality by developing a health indicator - the Realization of Potential Life Years (RePLY) - to adjust for unavoidable mortality risks. This approach, however, estimates unavoidable mortality risks by using the globally lowest mortality risks for each age-sex group of any country, and thus, benchmarking countries at different levels of development against the same unavoidable mortality risks without considering their heterogeneity. The current paper proposes to attempt to control for a country's national resources in estimating their (conditional) avoidable mortality risks. This allows the construction of a new health indicator - Realization of Conditional Potential Life Years (RCPLY). This paper presents and contrasts the empirical results for LY, RePLY and RCPLY based on life tables for 136 countries from year 2009.
\end{abstract}

Keywords: Mortality risk, avoidable deaths, longevity inequality, data envelopment analysis

JEL Classification: D6, I12 


\section{Introduction}

For any population, there are differences in health status amongst its people, and this is true even after controlling for 'natural' factors such as age and sex. Obviously natural factors go beyond just age and sex. Differences in genetic makeup and 'luck' may also cause disparities in health within a population. To the extent that these natural factors are outside the controls of individuals or society as a whole, the health inequality caused by them is arguably of lesser concern (Fleurbaey \& Schokkaert, 2009). From a policy perspective, the bigger concern is the health inequality that is avoidable and thus, potentially unfair. ${ }^{1}$ However, such policy dialogue is possible only if one can measure and distinguish avoidable and unavoidable health inequality.

Measuring health inequality within a population, even without distinguishing its avoidable and unavoidable components, is already difficult. A key reason is the lack of reliable data on individual health status, especially for developing countries. In the case that data do exist, like those on self-reported health ${ }^{2}$, they typically are only available for individual countries at sporadic years or they lack comparability across countries (Salomon et al., 2004). This makes comparisons of health inequalities across countries or over time very difficult. A health status measure that is relatively free from this problem is life years (LY) (or equivalently age-at-death). For any given population, in general, healthy people live longer and therefore have a greater LY. Based on this connection Le Grand (1987, 1989) first proposed using the inequality in the distribution of LY as a proxy for health inequality.

Using LY as an indicator of health status has several merits. Firstly, there is little ambiguity in deciding whether a person is alive or dead. Secondly, other things equal, the relationship between population health and LY is monotonic and health is a good predictor of

\footnotetext{
${ }^{1}$ Health inequality due to risky behaviors such as smoking and drinking could be considered as avoidable but not necessarily unfair if people conduct those activities at free will and with the full knowledge of the consequences. See Fleurbaey \& Schokkaert (2009) for a more detailed discussion.

${ }^{2}$ For instance, Allison and Foster (2004) use self-reported health status data from the US National Health Interview Survey to examine health inequality in the US.
} 
subsequent mortality (Van Doorslaer and Gerdtham, 2003). Thirdly, vital statistics are one of the mostly commonly collected demographic data, even in many developing countries. The publication of life tables, which standardize mortality statistics, further facilitates crosscountry and temporal comparisons of health status. Nevertheless, according to the World Health Organization (2013) "As of 2009, only $25 \%$ of the world population lived in countries where at least $90 \%$ of births and deaths are registered" (pp.20), although those countries with poor vital statistics are unlikely to have better quality data for other health measurements.

Using LY as a health indicator also has its limitations. Firstly, it may still suffer from bias if there is age misreporting, which is more common for older groups and developing countries (Preston et al 1999; Coale and Kisker 1986). Secondly, at the individual level it is uninformative about morbidity and thus a person who died at an old age but had suffered long-term illness may have been worse off than a person who lived a shorter but healthy life. In addition, reduced LY due to some causes of death may be a better proxy for proceeding morbidity than for other causes of death and thus the link between LY and health may change as countries develop. For instance, as a country grows and urbanizes, deaths due to behavioural problems such as suicide, car accidents, drinking and illicit drugs could displace traditional health impediments like malnutrition and infectious diseases; and the two types of mortality may have different preceding morbidity profiles (Benjamins et al., 2004). Because of these three limitations, the distribution of LY and its variants should be considered as an indicator of an important yet specific aspect of health inequality - longevity inequality (or, in equivalent, mortality inequality). Fourthly, and particularly relevant to this paper, LY does not distinguish between avoidable and unavoidable deaths. The very fact that every human is mortal is the strongest evidence that some mortality risks are unavoidable. Since unavoidable deaths, by definition, cannot be prevented by intervention, they have less immediate policy and resource implications than avoidable deaths, and the variation in LY caused by unavoidable mortality should not be considered as socially unjust. Tang, Chin and Rao (2008) 
develop an indicator focusing only on the age-at-avoidable-death in order to address this issue. Building on that effort, Tang, Petrie and Rao (2009a) further integrate the proportions of unavoidable deaths and the age-at-avoidable-death into a more comprehensive health indicator called the Realization of Potential Life Years (RePLY). ${ }^{3}$

The RePLY indicator measures people's LY as a proportion of their “potential” LY; therefore, its value is bounded between zero and one. Those people whose deaths are unavoidable, by definition, have lived their full potential LY and thus have a RePLY equal to one, and those people whose deaths are avoidable have not realized their full potential LY and therefore have a RePLY less than one. The numbers of avoidable and unavoidable deaths are estimated based on the probabilities at which the two types of deaths occur in each age-sex group. To analyse longevity inequality, RePLY can be used to replace LY to measure longevity on an individual basis and, hence, its distribution across the population. Since RePLY has filtered out the natural mortality differences between ages and sexes, it can be used to measure potential age or gender inequality in longevity that may be caused by unequal or inefficient allocation of resources, or by other socioeconomic, behavioural, cultural or environmental factors that increase mortality (Tang, Petrie and Rao, 2007).

The estimation of potential LY in Tang, Petrie and Rao (2009a) is based on the estimation of a "frontier profile" of mortality rates of 191 countries. This leads to the concept of a hypothetical reference or frontier country, whose mortality rates are used to proxy for the unavoidable mortality risks. The gap between the mortality rate of each age-sex group of a country and that of the frontier country is an indication of the country's excess or avoidable mortality risks for that group. It is postulated that if the country has the same amount of resources as the frontier country and uses it as efficiently, it could close the mortality gap. The use of a large cross-country dataset allows one to compare and contrast longevity inequality across both developing and developed countries. The drawback of this approach is that the

\footnotetext{
${ }^{3}$ For discussions on the RePLY measure, see Castelli and Nizalova (2011), Norheim (2010) and Rodriguez and Lopez-Valcarcel (2011).
} 
frontier profile of mortality rates is, as expected, determined by the mortality rates of mostly high-income countries. This means that the health performance of low-income countries is benchmarked against that of their affluent counterparts.

It is unrealistic, however, to expect that poorer countries have the same capacity to provide quality food, water and sanitation, shelter, and health care to their residents as richer countries. As such, the avoidable mortality gap currently estimated in the RePLY framework does not provide a good indication how much improvement these poor countries, themselves, could potentially achieve in the short run through more efficient usage or allocation of the resources at their disposal. The main focus of the current paper is, therefore, to develop a health indicator that takes into account the resource constraints faced by countries, and use it to measure longevity inequality within countries. To achieve this objective, we modify the RePLY measure. Specially, we estimate the frontier mortality profile using the Data Envelopment Analysis (DEA) method (Coelli, Rao, O’Donnell et al., 2005) attempting to control for the resources available to each country in the short run by considering their per capita incomes. This allows us to estimate a new measure of health status - Realization of Conditional Potential Life Years (RCPLY).

We estimate in total three health status measures, namely LY, RePLY, and RCPLY. Simply put, LY is a longevity indicator based on total mortality risks; RePLY attempts to distinguish between global avoidable and unavoidable mortality risks; and RCPLY attempts to distinguish between unconditional unavoidable (global), conditional unavoidable (local), and conditional avoidable mortality risks. A comparative analysis of the three measures can be used in gauging the importance of controlling for unavoidable mortality risks and resources respectively. For instance, if a country's average LY, RePLY, and RCPLY are equal to 64, 0.7 and 0.8 respectively, it means that the country's population on average live 64 years, which is equal to only $70 \%$ of their potential life years unconditional on their country's resources but $80 \%$ of their potential conditional on their country's resources. This means that 
only one-third of the $30 \%$ shortfall from their full potential could be explained by the country's lack of resources, with the remaining two-thirds by non-resource related factors such as institutions, culture, environment, and, most noticeably, inefficiency in resource allocation and utilization.

The rest of the paper is organized as follows. Section 2 explains the data used in the empirical work. Section 3 reviews the concepts of unavoidable mortality risk and RePLY. Section 4 explains the concept of RCPLY and how it can be constructed using the DEA method. Section 5 briefly introduces the DEA method. Section 6 discusses measuring health inequality when the health variable is bounded, as in the case of RePLY and RCPLY, and to a lesser extent, LY. Section 7 reports and discusses the findings, and Section 8 concludes.

\section{Data}

The analysis in this paper requires mortality and income data. Mortality data are drawn from the 2009 life tables of 136 countries (World Health Organization, 2012). The sample coverage is constrained by the limitation of income data. Countries with a population less than one million are excluded due to the concerns that these countries may have large variability of observed mortality risks, which could lead to bias in the estimation of the frontier mortality rates.

Life tables provide information on the estimated probability of death in each age-sex group and subsequently the number of deaths for a stationary population. As a result, the calculation of longevity inequality in this study is based on the stationary population rather than the actual population. ${ }^{4}$

The income data is drawn from the World Development Indicators database. Income is measured by real GNI per capita (PPP, current international dollar). ${ }^{5}$ We use GNI capita instead of GDP per capita as a measure of income because the former takes into account of

\footnotetext{
${ }^{4}$ The actual population can be used to scale up the stationary population if one is interested in measuring average health or health inequality for a multi-country region or the world as a whole.

${ }^{5}$ We do not use constant international dollars because of the lack of data.
} 
net factor income (i.e. income earned overseas net of income paid to foreign countries), which can be important for countries that have large number of workers working overseas (e.g. the Philippines) or large foreign liabilities (e.g. Australia). We use year 2004 income data to mitigate possible reverse causality from health to income (to be explained in detail in Section 4). We also apply the method to year 2000 life tables and, correspondingly, year 1995 income data for robustness tests and to explore the stability of these measures over time.

\section{Unavoidable Mortality Risks and Realization of Potential Life Years (RePLY)}

Avoidable and unavoidable mortality risks in this section all refer to the unconditional risk, so we omit the term "unconditional" till the next section for ease of expression.

\subsection{Reference Distribution of Unavoidable Mortality Risks}

Mortality risks are not static; they can be affected by genes, behaviour, resources, technology, socioeconomic factors, and the environment. The effects of these factors are not independent of each other. For instance, the fact that the mortality rate is age and sex dependent is evidence of the effects of genes and behaviour; however, technology, such as vaccinations and medication, can mitigate those effects to various degrees. Resources, such as, food, education, shelter, law and order can also reduce mortality risks. Furthermore, while exposure to different types of environmental factors, such as cold weather and heat waves, could lead to very different mortality risks, people could be shielded from these environment risks when sufficient resources and appropriate institutions are in place (Tang, Petrie and Rao, 2009b; Tang, Chin and Rao, 2008).

For a given level of technology, no matter the resources available, some mortality risks, like those related to genes and chance, cannot be eliminated. These mortality risks can be classified as unavoidable mortality risks. The gap between the actual mortality risks and the unavoidable mortality risks equals the avoidable mortality risks. Avoidable mortality is a long-standing notion in the health literature. Yet, the method to determine whether a death is 
avoidable or unavoidable is still a contentious issue. For instance, in the calculation of potential years of life lost (PYLL) typically an upper bound of age 70 is used (e.g. Romeder \& McWhinnie 1977), implicitly assuming that all deaths before age 70 are avoidable and all after are unavoidable. On the contrary, the Centers for Disease Control and Prevention of the United States (CDC 1986) classifies deaths caused by violence, starvation, consumption of tobacco, poor diet and physical inactivity (i.e. obesity), alcohol consumption, toxicants, illicit use of drug, and vehicle accidents as preventable. A limitation of this approach is that it identifies only a subset of avoidable deaths and noticeably excludes all disease related deaths. An extension of this approach is therefore to list the types of health conditions, such as influenza or drowning, as avoidable. This approach was first suggested by Rutstein, Berenberg, Chalmers, et al. (1976); for a recent survey, see Nolte \& McKee (2004), Kossarova, Holland, Nolte and McKee (2009), and Castelli and Nizalova (2011).

An alternative approach is to set the actual mortality rates of a group, typically a country or province with a very high life expectancy, as the reference (i.e. unavoidable) rates to measure the excess (i.e. avoidable) mortality of the others. This approach has a long tradition, starting with Farr (1885), and then being adopted by Woolsey (1981), Uemura (1989), McCracken (2002), and, most recently, by Tang, Chin and Rao (2008). In Farr and McCracken, regions with the highest socioeconomic status are chosen as the reference group. A shortcoming of this approach is that a single region is unlikely to have the lowest mortality rate for all age-sex groups. Woolsey (1981), Uemura (1989), and Tang, Chin and Rao (2008) circumvent this problem by constructing the reference unavoidable mortality rates using data from multiple regions or countries. Amongst all these studies, Tang, Chin and Rao (2008) is the only one that uses international mortality data that cover countries at all levels of income and development -191 countries in total. Using a data envelopment method ${ }^{6}$, Tang, Chin and Rao (2008) construct a hypothetical frontier country that has the lowest mortality rates for

\footnotetext{
${ }^{6}$ This method is like the DEA method used in this paper, except that it does not involve any input and production function.
} 
each of the age-sex groups. The mortality rates of a frontier country are then used to estimate the unavoidable mortality rates.

The method used by Tang, Chin and Rao (2008) is as follows. Suppose there are $K$ countries and the probability of a person of sex $s$ in country $k$ who survives to age $x$ will die before reaching the next birthday is denoted by $q_{x s k} \cdot{ }^{7}$ Let $\tilde{q}_{x s}$ be the probability of dying for a person of the same age and sex in the hypothetical frontier country. Then, $\tilde{q}_{x s}$ is defined as

$$
\tilde{q}_{x s}=\left\{\begin{array}{cr}
\min _{k}\left\{q_{x s k} ; k=1,2, \ldots K\right\} & x<100 \\
1 & x \geq 100
\end{array}\right.
$$

where all deaths at ages greater than 100 are considered unavoidable for simplicity.

\subsection{Realization of Potential Life Years (RePLY)}

Tang, Petrie \& Rao (2007) employ the measures of avoidable and unavoidable mortality risks derived using the Tang, Chin and Rao (2008) method to develop the Realization of Potential Life Years (RePLY). The RePLY is defined as the ratio of a person's actual LY compared to his potential LY. For an avoidable death that occurs at age $x$ of sex $s$, the person has not fully realized his potential LY. If the person had been born in the frontier country, he/she would be expected to live till $x+\tilde{e}_{x s}$, where $\tilde{e}_{x s}$ is the life expectancy for an identical person in the frontier country, constructed from the series of $\left\{\tilde{q}_{x s}\right\}$ using standard life table methods. ${ }^{8}$ In contrast, for an unavoidable death at any age, the person has lived 100\% of their potential LY that nature and current technology permitted (i.e. additional resources would not have made the person live longer). Therefore, the RePLY measure associated with each observed death at age $x$ of sex $s$ can be expressed as:

\footnotetext{
${ }^{7}$ Here $q$ is a conditional probability as it is conditional on the person having survived from birth till age $x$. However, we simply use the term "probability" rather than "conditional probability" throughout the paper so that we can preserve the word "conditional" for cases where the probabilities are measured after controlling for income.

${ }^{8}$ In life tables, life expectancy at age $x$ in country $k, e_{x k}$, is defined as the number of further years a person is expected to live if the person has lived to age $x$.
} 


$$
\operatorname{RePLY} Y_{x s}=\left\{\begin{array}{cc}
1 & \text { for an unavoidable death } \\
\frac{x}{x+\tilde{e}_{x s}} & \text { for an avoidable death }
\end{array}\right.
$$

This means that RePLY can be used to measure longevity inequality within as well as between age-sex groups.

\section{Conditional Unavoidable Mortality Risks and Realization of Conditional Potential}

\section{Life Years (RCPLY)}

\subsection{GNI per capita as a measure of national resources}

The assumption of unavoidable mortality risks being country invariant is based on the assertion that unavoidable mortality risks are driven by, besides genes, globally available technology. A limitation of this assertion in practice is that even though technology is globally available, its purchase and adoption is resource dependent. For instance, poor countries are typically in great need of even basic medical supplies and personnel. As a result, the estimates of avoidable mortality rates based on the global frontier of unavoidable mortality rates often formed by the richest countries are only a global concept with limited policy relevance for developing countries in the short to medium term.

In this paper we propose to estimate avoidable mortality risks after controlling for country-specific resources as estimated by income. The rationale and limitation of using income as a measure of health-related resources warrants careful discussion. Obviously, income is not the only dimension of health-related resources. Numerous studies suggesting that non-income factors contributed greatly to the global trend of rising life expectancy, especially the diffusion of health technology from advanced to less advanced countries. For instance, Caldwell (1986) discusses at length how some low-income countries could achieve high life expectancy by mobilizing their limited resources into health and education. Preston (1975) estimates that increases in income itself accounted for about 10-25\% of the rise in life 
expectancy for the world as a whole between 1930 and 1960. However, recently Mackenbach and Looman (2013) revisit Preston's thesis and find that after 1960, the declines in European mortality from cardiovascular disease - one of the major cause of deaths in Europe - were mainly a result of higher income. In other words, their findings suggest that the contribution of economic growth to mortality reduction has become much larger in recent decades.

Against this background, it is important to distinguish between the global trend of falling mortality rate over time on the one hand, and differences in mortality rates across countries at a given point in time on the other. At any given point in time (and thus with a given set of technology), there is a clear logistic relationship between life expectancy and income across countries - the Preston curve. This relationship is remarkably resilient amid the global trend of falling mortality risks over time. Figure 1 is a plot of the Preston curves for 1980 and 2010 respectively, using GNI per capita as a measure of income. Both curves look essentially the same as that for 1930 as reported by Preston (1975). In fact, when we fit a logistic regression model to the data, log income has a slightly higher 'explanatory power' over the life expectancy differentials across countries in $2010\left(R^{2}=0.74\right)$ than in $1980\left(R^{2}=\right.$ 0.70). Similar to what Preston concluded based on much older data, these recent data demonstrate that, even if changes in income is not necessarily the most important factor in determining the changes in mortality rate over time, income differential can explain a large proportion (e.g. $74 \%$ for 2010 data) of mortality differential across countries at any given period. In addition, income is usually highly correlated with those non-income factors that impact on health, making income arguably the best single proxy for those factors. Therefore, focusing on income-related resources in cross-country analyses like the current paper provides a reasonable starting point.

As pointed out by Bloom and Canning (2009), the relationship between national income and health are bi-directional in that, while income growth may improve health, health improvement can also boost economic growth. This raises the issue of whether we should 
interpret the Preston curve as evidence of the income-to-health pathway or the other way round. Methodologically speaking, it is very difficult, if not impossible, to determine the direction of causality using cross sectional data. However, if the Preston curve is predominantly a result of the health-to-income effect, then the well-fitting logistic functional form in Figure 1 would imply that life expectancy has increasing marginal returns to income. On the contrary, if the curve is predominantly a result of the income-to-health effect, then its logistic functional form would imply that income has diminishing marginal returns to life expectancy. The former scenario is much less plausible. This is because, as many highincome countries have already had very low mortality rates for young and middle-age groups, further increment in life expectancy has to come from the reduction in mortality rates for the old-age group. Given the elderly population has largely exited from the labour market, it is hard to see how their longevity will be translated into higher productivity - an ultimate source of higher income. As such, it is unclear that cross sectional data in the Preston curve captures much of the health-to-income effect. In the empirical analysis, we use a lagged income measure to further reduce the potential bias.

While the income differential alone can explain nearly three-quarters of life expectancy differentials across countries, there is still one-quarter of variations left unexplained. This raises the question of whether one should control for other determinants of mortality differentials across countries besides income that are outside a country's short-term control in the construction of the frontier mortality rates. Although this is operationally possible, in the context of DEA it adds additional complexities. When controlling for income, essentially we group countries by income level and compare a country with other countries with similar income levels - known as its peers. Suppose mortality risks are also affected by education and climate, and further that education is highly correlated with income while for climate perhaps less so. In this case controlling for education in additional to income will add little to the outcome. On the contrary, controlling for climate could change the outcome for 
some countries substantially, but that means that we now group countries together only if they have similar income levels as well as similar climates. For any given country, its number of peers will be smaller than or equal to before, and the smaller the peer group the more likely a country will become the top performer of its group. When a large number of mortality determinants have been controlled for, it is possible for each peer group to have only one country, and as a result, all countries would have zero (conditional) avoidable mortalities and thus no (conditional) avoidable mortality inequality at all. Yet, this is merely an artefact of having too few countries against which to benchmark each particular country.

The above discussion points out a key difference between modelling mortality risks and controlling for certain factors when measuring mortality inequality. In the case of modelling mortality risks, the purpose is to attribute as much of its variations (over time or cross country) to variations of external factors as possible. In the case of controlling for certain factors when measuring mortality inequality, the purpose is to find a meaningful benchmark for each country. What benchmark is meaningful depends on the problem at hand and in the current paper, we argue that it is meaningful to benchmark each country against those with similar amounts of resources and that income is a good proxy for this. However, if a researcher wants to examine climate-controlled mortality inequality, then she may choose to condition on malaria or tropical indicators. As such, we are not suggesting that RCPLY has to be conditional on one particular variable, but suggesting that if one wants to do so, how it can be done and how that may change the outcome as compared to the unconditional version RePLY. In that sense, the validity of RCPLY is very much dependent on whether the conditioning variable (e.g. income) is a good indicator of the external factors we want to control for (e.g. resources).

All in all, based on theoretical and practical considerations, in this paper we use only income to indicate the amount of resources available to each country. When the mortality risks of a country are benchmarked against the mortality risks of the best performing countries 
regardless of their income levels, we obtain the original RePLY; and when benchmarked against those of similar income levels, we obtain our new measure-RCPLY.

\subsection{The frontier approach to the determination of conditional and unconditional mortality risks}

This section describes the frontier approach to determine conditional and unconditional mortality risks for different age-sex groups. Let the survival probability of a person of age $x$, sex $s$ and living in country $k$ reaching the next age bracket be $p_{x s k}$, which is equal to one minus the probability of death (i.e. $p_{x s k}=1-q_{x s k}$ ). The probability is affected by, amongst other things, the country's income level $\left(y_{k}\right)$. As a result, some countries with higher income might attain a higher survival probability than country $k$. However, some other countries with the same income level as $y_{k}$ might also attain a higher survival probability. We illustrate these scenarios in Figure 2, which is a scatter plot of the survival probability of males aged 75-80 against log income for the 136 countries in our dataset. The value of, say, 0.61 for India means for that those Indian males who have survived to the age of $75-80$ on average have $61 \%$ chance of reaching 81 or higher ages. The solid line in Figure 2 is the frontier function that shows the maximum feasible survival probability for a country with a given income level. The frontier function is constructed using the DEA method, which is explained in Section 5.

Suppose country $k$ locates at point A (which is actually Malawi). The observed survival probability $p_{x s k}$ is equal to distance OA. The maximum feasible survival probability conditional on its income is given by:

$$
\hat{p}_{x s k}=f_{x s}\left(y_{k}\right)
$$

where $f_{x s}$ is the frontier function and on Figure 2, $\hat{p}_{x s k}$ is given by distance OB.

The maximum feasible survival probability with given technology, $\tilde{p}_{x s}$, is equal to distance $\mathrm{OC}$ and it is unconditional on income. Thus, all the observed survival probabilities, 
$p_{x s k}$, and maximum conditional survival probabilities, $\hat{p}_{x s k}$, are below or equal to the maximum feasible level $\tilde{p}_{x s}$. By definition, we have $p_{x s k} \leq \hat{p}_{x s k} \leq \tilde{p}_{x s}$, which implies $q_{x s k} \geq \hat{q}_{x s k} \geq \tilde{q}_{x s}$. The mortality risk $\hat{q}_{x s k}$ is the lowest mortality risk achievable in country $k$ given its income level $y_{k}$, while the mortality risk $\tilde{q}_{x s}$ is the lowest mortality risk achievable with the current technology regardless of income and is also defined in this study as the unconditional unavoidable mortality risk (distance CD). The difference between the lowest mortality risk possible conditional on income and the lowest mortality risk possible regardless of income $\left(\hat{q}_{x s k}-\tilde{q}_{x s k}\right)$ is defined in this study as the conditional unavoidable mortality risk (distance BC). From the policy perspective, given a country's income level, the conditional avoidable mortality risk (distance $\mathrm{AB}$ ) is what the country could potentially eliminate by better resource allocation and management. In other words, the conditional avoidable mortality risk is an indication of local, non-resource factors that impact on mortality rates.

The RCPLY measure associated with each observed death at age $x$ of sex $s$ is expressed as:

$$
R C P L Y_{x s k}=\left\{\begin{array}{cc}
1 & \text { for an unconditional unavoidable death } \\
1 & \text { for a conditional unavoidable death } \\
\frac{x}{x+\hat{e}_{x s k}} & \text { for a conditional avoidable death }
\end{array}\right.
$$

where $\hat{e}_{x s k}$ is the life expectancy of the hypothetical "local frontier country" and is constructed from the series of $\left\{\hat{q}_{x s k}\right\}$.

When benchmarked against the hypothetical local frontier country, the number of conditional unavoidable deaths, $U_{x s k}^{C}$, is given by

$$
U_{x s k}^{C}=N_{x s k}\left(\hat{q}_{x s k}-\tilde{q}_{x s k}\right)
$$


The number of conditional avoidable deaths for the group, $A_{x s k}^{C}$, is equal to the number of all deaths, $D_{x s k}$, minus that of the unconditional unavoidable deaths and conditional unavoidable deaths, $U_{x s k}$ :

$$
A_{x s k}^{C}=D_{x s k}-U_{x s k}^{C}-U_{x s k}=N_{x s k} q_{x s k}-N_{x s k}\left(\hat{q}_{x s k}-\tilde{q}_{x s}\right)-N_{x s k} \tilde{q}_{x s}=D_{x s k}\left(1-\hat{q}_{x s k} / q_{x s k}\right)(6)
$$

There are a number of reasons why conditional avoidable mortality may exist at certain age-sex groups. First, a smaller amount of health-related resources may be devoted to this age-sex group compared to that allocated by other countries with similar amounts of total resources. Second, these health-related resources may not be used as efficiency as other countries with similar amounts of resources. For instance, the benefit-to-cost ratio is typically high for preventative measures like vaccination and low for curative measures like surgeries; therefore, countries that do not invest sufficiently in preventative measures would be considered as having lower efficiency than others. Third, certain age-sex groups may engage in more risky behaviours like binge drinking than their peers in other countries. Forth, there could be institutional or cultural discrimination against certain segment of the population in terms of accessing to education and health, such as young females in developing countries.

\section{Constructing the Frontier using the Data Envelopment Analysis (DEA) Method}

The critical step in constructing RCPLY is to identify the frontier for each age-sex group. The implicit assumption is that there is a functional relationship between survival probability and income, which implies that the state of health technology helps transform resources into survival probability. This function, like production technology, shows the maximum attainable survival probability. The DEA method constructs this frontier using a piecewise linear frontier that envelops all the data points similar to the one drawn in Figure 2. The method is to the construction of the frontier that identifies the maximum feasible survival probability, $\tilde{p}$, for a given country with a given per capita income, $y$. When $y$ and $p$ are observed for a large number of countries, the DEA method identifies a benchmark (i.e. the 
best performance) against which the actual survival probability in a given age group can be compared. A full description of the DEA method and its application can be found in Coelli, Rao, O’Donnell et al. (2005) but a brief description is provided as supplementary material to this paper.

Once the frontier is identified, we can proceed to find the maximum possible survival probability at a given income level which is used in defining the conditional unavoidable mortality risk as shown in Figure 2. Points on the envelope (solid line) define the best practice for countries with given levels of income. Amongst the 136 countries in our dataset, four top performing countries highlighted as solid black dots for 75-80 year males, namely Switzerland, Ecuador, Nigeria, and the Democratic Republic of the Congo (DRC) in descending order of income. These countries, by definition, have zero conditional avoidable mortality risks for this particular age-sex group, and all other countries' mortality rates are benchmarked against combinations of the mortality rates of these "peers" or best performing countries. Amongst these four countries, Switzerland has the highest survival probability; therefore, it is the only country that has zero conditional and unconditional avoidable mortality risks for this age-sex group.

DRC is a special case as it sits on the "edge" of the frontier because it has the lowest income level (\$240 in year 2004) amongst all the countries in the dataset. Due to having the lowest income, even if its survival probability were to substantially drop, it would remain on the frontier. In fact, this is also the case for all other age-sex groups, making DRC the only country that has zero conditional avoidable deaths for all age-sex groups and, thus, perfect conditional health equality! Since this is an artifact of DRC not having a peer against which to benchmark its own performance, no meaningful conclusions can be draw about DRC. Despite this, DRC is kept in the estimation; otherwise, the same situation would hold for the country with the second lowest income level in the sample. 
Using the same procedure we obtain lists of countries that have zero conditional avoidable mortality risks for all other age-sex groups. The results are reported in Table 1, where countries are listed according to their incomes. For instance, for new born males (i.e. age 0), those in Sweden, Slovenia, Estonia, Sri Lanka, Viet Nam, Eritrea, and DRC have the lowest mortality rates and thus the highest chances to survive till age 1 amongst the countries in their respectively income groups; for males that survive to age 1, those in Finland, Malaysia...Eritrea, and DRC have the lowest mortality rates and thus the highest chances to survive till age 5 amongst the countries in their respectively income groups; and so forth. The countries in bold are those with the lowest mortality risks for the various age-sex groups in our dataset and therefore also provide the benchmark mortality for the construction of RePLY.

\section{Inequality Measures}

Once we obtain the estimates of LY, RePLY and RCPLY for each age-sex group, we can assess the degree of mortality inequality within a country. Due to the nature of life tables, we can only stratify the population by age and sex, and, in the case of RePLY and RCPLY by avoidable/unavoidable deaths as well.

There are numerous inequality indicators used to summarise the distribution of health, and these indicators embody different value judgements about what change in health would constitute an increase in inequality. In this paper, we use a number of commonly used inequality measures to illustrate and test the robustness of the results to the choice of inequality measure. In particular we employ the Gini coefficient in health (relative inequality in terms of health), the Gini coefficient in health-shortfall (relative inequality in terms of health-shortfall), the Erregyers index (an absolute inequality index) and the Wagstaff index (a combination of relative inequality in health and relative inequality in health-shortfall). ${ }^{9}$

The Erregyers and Wagstaff indices are two recently proposed indices for health outcome measures with bounded values. The Erreygers index (Erreygers, 2009) is equal to the

\footnotetext{
${ }^{9}$ The formulas for these indexes are provided as supplementary material to the paper.
} 
generalised Gini coefficient standardised by the maximum inequality possible given the bounds of the health variable. While the Wagstaff index (Wagstaff, 2005; Erreygers, 2009) is the Gini coefficient standardised by the maximum inequality possible given the bounds of the health variable and the mean level of health. The Wagstaff index adopts similar properties as the Gini coefficient in health, the Gini coefficient in health-shortfall, and the Erregyers index when mean health is respectively very low, very high, close to the midpoint between the upper and lower bounds (Allanson and Petrie, 2013; and Kjellsson and Gerdtham, 2013).

These four inequality indexes are all rank-dependent measures where their differences only lie in how they react to absolute and relative changes in health in the population (Allanson and Petrie, 2012). For instance, an increase in health by the same absolute amount (e.g. one more LY) in the whole population is considered inequality preserving for the Erregyers index while that by the same proportion (e.g. 1\% higher in LY) is inequality preserving for the Gini coefficient in health. In addition we also derive two non rankdependent measures, the Theil $\mathrm{T}$ and Atkinson index (with the "inequality aversion" coefficient set to 0.5 ) in health, which are similar to the Gini coefficient but provide alternative weighting schemes.

\section{Empirical Results}

\subsection{Cross-country comparison of health status}

We first look at the results for average RePLY and RCPLY for all 136 countries in our dataset. The results are reported in Table 2 . There is large variation in longevity across the countries in that one of the best preforming countries, Japan, has an average LY nearly 36 years (or $75 \%$ ) longer than that of the poorest performing one, Malawi. Even if RePLY is used to distinguish avoidable and unavoidable mortalities, Japan remains the best preforming country overall ${ }^{10}$, with its population realizing $98.9 \%$ of its potential life years. Malawi also remains the worst performing country with a realization rate of $56.8 \%$. The huge health gap between the two

\footnotetext{
${ }^{10}$ Japan, however, does not have the highest survival probabilities for some age-sex groups.
} 
countries should not come as a surprise given Japan is 49 times as rich as Malawi, and thus, has far more resources to invest in health. As such, neither LY nor RePLY can be used to indicate how much of Malawi's poor longevity performance may be due to its lack of resources versus non-resource factors, such as but not limited to, inefficient allocation and usage of the resources currently at its disposal.

The new measurement, RCPLY, provides a tool to answer this question as it controls for income differences across countries. According to RCPLY, the Japanese population on average is found to have realized $99.0 \%$ of its conditional potential LY, while Malawi $73.0 \%$. This means that, by being one of the richest countries in the world, Japan's longevity performance is one of the very best in the world or even amongst the high-income countries. On the other side, even after taking into account its income level, Malawi still has $27.0 \%$ (= $100 \%-73.0 \%$ ) of its potential LY yet to be attained. This means that other countries of similar incomes as Malawi are significantly out performing it. This also suggests that, of the $43.2 \%(=100 \%-56.8 \%)$ shortfall of its potential LY, as much as five-eighths $(27.0 / 43.2=$ 5/8) can be attributed to non-income factors, and the remaining three-eighths explained by the country's lack of resources.

This example therefore illustrates how RCPLY can be used in conjunction with RePLY to distinguish between resources and non-resource factors in explaining health shortfall. This is particularly useful when assessing how important resource factors are in determining the health performance of developing countries, as poor economic conditions can easily mask poor health system efficiency.

\subsection{Cross-country comparison of longevity inequality}

As explained earlier we consider in total six inequality indexes. Given the current literature does not have conclusive evidence which of them is the preferred one for health inequality measurement, we first present the general findings based on all six indexes before narrowing 
down our focus. For each of the 136 countries in the dataset, we apply each of the six inequality indexes to estimate the dispersion of attainment of conditional potential life years (i.e. RCPLY) amongst the 44 age-sex groups. Some of the resulting inequality index values are highly correlated while others are not. Table 3 reports both the Pearson and rank correlation coefficients for these indexes. The Gini coefficient, Erreygers index, Theil index and Atkinson index are highly correlated with each other, as evident in their Pearson correlation coefficients being higher than 0.95 and rank correlation coefficients higher than 0.94. On the contrary, the Wagstaff index has very low correlation with any of the other indexes, while the Gini coefficient for shortfall is moderately negatively correlated with the others.

Both the Wagstaff index and Gini coefficient for shortfall have negative correlation with other indexes. As noted in Section 5 the Wagstaff index acts similar to the Gini coefficient in shortfall when the mean health approaches the upper bound and for the health measures considered in the current paper. When mean health is close to the upper bound of health an increase in health in terms of relative shortfall is very different from an absolute increase or relative increase in terms of health (see Allanson and Petrie, 2012). In particular the Gini coefficient in shortfalls and the Wagstaff index are sensitive to the minority that falls behind and, thus, embody very different value judgments from the other inequality measures. The results in Table 3 show that the Erreygers index is highly correlated to all other indexes except for the Wagstaff index. Therefore, in what follows we focus on the Erreygers and Wagstaff indexes only as representative cases.

In Table 4 we examine the relationship between the national average longevity and longevity inequality. When the Erreygers index is used, longevity inequality is found to be highly correlated with average longevity for all LY, RePLY, and RCPLY. This is because, for our dataset, average longevity provides a strong indicator of the absolute longevity differences and thus dominates the Erreygers measures of longevity inequality. The correlation between 
the Wagstaff measures of longevity inequality and their corresponding average longevity measures are much smaller. This is because, once again, the Wagstaff index is sensitive to the performance of the minority population - those who suffer from avoidable mortality. The correlation between average longevity and longevity inequality measures based on RCPLY (0.294) is smaller than that based on RePLY (0.467), which in turn is smaller than that based on LY (-0.629) in absolute terms. This is because at each stage the variation due to certain unavoidable mortality is being removed so that only avoidable differences are focused on. With mortality we see that a large proportion of individuals differences come down to chance. Therefore, to see more clearly the differences that are potentially "unfair", one may wish to remove these unavoidable differences, and we propose RePLY and RCPLY as useful tools to achieve that.

\subsection{A case study on China and Russia}

To further demonstrate how the new measure RCPLY can contribute to analysis of longevity and gender inequality in longevity within a country, we select China and Russia from the 136 countries for more detail discussion. Table 5 reports average longevity respectively for the total population, male population, and female population for each country.

China's per capita income in 2004 is equal to $\$ 3590$ and Russia's income is nearly three times as large at $\$ 10,030$. Despite its much higher income level, Russia's life expectancy at birth (i.e. average LY) is six years shorter than China's and its population attains $81.4 \%$ of its potential LY (i.e. RePLY) on average, compared to $88.5 \%$ for China. However, once we take into account their respective income levels, Russia's population has only marginally increased its attainment of potential life years to $84 \%$ (i.e. RCPLY), while China has substantially increased its to $94.7 \%$. This means that, on average, what separates Russia from the best preforming countries is not the lack of resources, but some non-resource related factors, which could be its low efficiency in allocating and utilizing resources for 
health promotion or adverse lifestyles of its population (Zaridze et al. 2014). On the contrary, for China, resources and non-resource factors are roughly equally important in extending the LY of its population.

Although on average China has very good longevity given its income level, a closer look at the outcomes for individual age-sex groups reveals a more complex picture. Figure 3 shows the plots of the average RePLY and average RCPLY for each age-sex group for the two countries. Since RCPLY is a conditional measure, the average RCPLY for any age-sex group must be at least as large as the average RePLY. Only if a country has the lowest mortality for a particular age-sex group or if a country's income is greater than the income of the best performer should the two measures be the same. Consistent with the national outcome, controlling for income sees the average realization of potential LY for all age-sex group increase substantially for China. However, the extent of increase is highly uneven across different population groups. In particular, for the 15-20 years old male population, the average RCPLY (0.39) is not much larger than its average RePLY (0.33). This means that, some countries with an income level comparable to China were able to achieve a survival probability for this age-sex group close to (but still smaller than) the world's best performing countries. Another way to interpret this result is that, this age-sex group in China faces less favorable conditions as compared to its peers (countries with the best outcomes) of similar income levels. Furthermore, the average RCPLY (0.48) for Chinese female infants is far smaller than that for their male counterparts. This means that, even compared to countries of similar income levels, China still has much room to improve on the health of its female infant population.

The RePLY and RCPLY outcomes for Russian males and females, in comparison, are much more similar. But there are still noticeable differences between the two measures for infants. What this means is that both gender groups face more or less equally unfavorable conditions beyond the lack of resources. 
The contrast between the male and female results in Figure 3 highlights the relatively larger pro-male gender inequality in longevity in China. Many longevity measures like LY cannot be directly compared across the two sexes because of their genetic differences. However, since RePLY and RCPLY both have controlled for the potential LY for the two sexes, which are affected by genes amongst other factors, the two measures can be used as a measure of gender inequality in longevity resulting more from non-genetic factors, as in Figure 4.

Figure 4 is a plot of the ratios of male to female average RePLY and average RCPLY for China across different age groups. A ratio higher than one means pro-male longevity inequality. The figure shows that, if we compare China with the best forming countries in the world regardless of their income levels, using RePLY, China exhibits strong inequality against female infants, but the inequality by and largely gradually reduces with age. This finding is consistent with the well-known fact that there is strong preference for males in China, especially in rural regions. Indeed, there have been reports and studies of the "missing girls" phenomenon in China (e.g. Johansson and Nygren 1991; Cai and Lavely 2003), highlighting the inequality against female infants. However, gender inequality against females declines with ages and even reverses for certain age groups. This picture largely remains the same when we compare China with its peers of similar income using RCPLY. One difference however is that the gender inequality in longevity in favor of males increases dramatically from the $0-1$ age group to the 1-5 one. This means that, amongst its peers of similar income level, Chinese males aged 1-5 performs much better than their female counterparts. While male infant mortality rates in China are very close to their peers the female mortality rates are still a long way off their peers in relative terms.

In Figure 5, we further examine how the RCPLY outcomes for China and Russia change from year 2000 to 2009 to examine the stability of these measures over time. The figure shows that while the average RCPLY for some age-sex group increase over time, 
others decrease. As technology advances and economies grow over time, the health condition for every age-sex group in a typically country should improve in absolute terms. When such improvement takes place in the frontier countries, it raises the potential LY against which other countries' actual LY are measured. Also, even if the global technology were static over time, if a country's income grows faster than its peers, over time it will progress to a higher income group and therefore be compared to different peers. Because of these, it is possible for the average RCPLY of an age-sex group of a country to improve or deteriorate over time. The figure also shows that the improvement of average RCPLY for many age-sex groups is bigger for China than for Russia. This suggests that although China has developed quickly in terms of income, its economic advancement has also been matched by substantial improvements in health outcomes, while for Russia its RCPLY (relative position) is rather stagnant for all agesex groups over this period.

\section{Concluding Remarks}

The current paper represents another stage in improving the measurement of health status and associated health inequality indicators, especially for developing countries. This research started with an attempt to improve on a "classic" indicator, life years (i.e. age-at-death), which resulted in the development of age-at-avoidable-death. The methodology was subsequently used in developing the RePLY indicator. The current paper proposes an improved measure, namely RCPLY. In each evolution, additional factors and complexities are controlled for. From LY to age-at-avoidable-death, we considered the differences between avoidable and unavoidable deaths and examined the inequalities in the age-at-avoidable-deaths; from age-atavoidable-death to RePLY, we have controlled for the differences between avoidable and unavoidable deaths by combining both in a single measure; from RePLY to RCPLY, we have controlled for the differences in available resources across countries so that differences in LY 
due to resource constraints are removed. ${ }^{11}$ Therefore, RePLY can be used to measure average health outcome and inequality associated with both resources and non-resource factors, while RCPLY can be used to measure those associated mainly with non-resource factors, such as inefficiency in resources allocation and usage. As such, RCPLY-based measures offer countries a target that is achievable in the short to medium terms with the resources at their disposal, while RePLY-based measures offer a higher, and thus, longer-term target.

In the current paper, we use RePLY and RCPLY to examine age and gender inequality in longevity in China and Russia. A merit of using these indicators is that they can presumably remove gene-related factors and therefore allow researchers to isolate other nonnatural causes of gender inequality in longevity. The method can also be used to study racial inequality in health; for instance, Tang and Rao (2008) study longevity inequality between Black and White populations in the US and Maori and non-Maori groups in New Zealand using age-at-avoidable-death. The current paper also demonstrates the role of RCPLY in measuring longevity inequality in 136 countries using a range of inequality indexes. An important merit of the RCPLY measure is that it provides a method to consider the performance of a country against others while controlling for country specific factors, which may affect its performance, and therefore offers a meaningful tool for international comparison of health status and inequality.

Lastly, two caveats are in order to highlight future directions of research. When only income is controlled for in constructing RCPLY as done in the current paper, it is not feasible to pinpoint which non-resource factors give rise to the mortality gap between a country and its peers. However, this does not diminish the relevance of the new measure as it allows quantitative monitoring of a large number of countries using comparable data. It also indicates problematic population groups to be investigated with more analytic studies aiming at the

\footnotetext{
${ }^{11}$ In Petrie and Tang (2014), we achieve the same by developing an indicator that focuses on health-shortfall instead of health-attainment as in the current paper.
} 
identification and removal of the causes that produce mortality disadvantage for the country in concern. $^{12}$

Like any other mortality measures, RePLY and RCPLY omit morbidity and do not distinct different causes of death. This is largely because, at the moment, data on morbidity or on the mortality rates of different causes of death are not sufficiently detail at the country-agesex level. Rectifying this problem requires concerted efforts on data collection by national and international institutions.

\footnotetext{
${ }^{12}$ We would like to thank a reviewer for clarifying this.
} 


\section{References}

Allanson, P. \& Petrie, D. (2013). 'On the choice of health inequality measure for the longitudinal analysis of income-related health inequalities' Health Economics, vol. 22, no. 3, pp. 353-65.

Allanson P. \& Petrie, D. (2014). "Understanding the vertical equity judgements underpinning health inequality measures," Health Economics, forthcoming.

Alesina, A, Devleeschauwer, A, Easterly, W, Kurlat, S \& Wacziarg, R (2003). 'Fractionalization' Journal of Economic Growth, vol. 8, pp. 155-194.

Allison, RA, \& Foster, J (2004). 'Measuring health inequality using qualitative data' Journal of Health Economics, vol. 23, no. 3, pp. 505-524.

Benjamins, M. R., Hummer, R. A., Eberstein, I. W., \& Nam, C. B. (2004). Self-reported health and adult mortality risk: an analysis of cause-specific mortality. Social Science \& Medicine, 59(6), 1297-1306.

Bloom, D \& Canning, D (2009). 'Population health and economic growth', in M Spence and M Lewis (ed.) Health and Growth, Chapter 3, p.53-76 World Bank Publication, Washington, D.C.

Barro, RJ \& Lee, J-W (2001). 'International Data On Educational Attainment: Updates And Implications,' Oxford Economic Papers, vol. 53, no. 3, pp. 541-563

Cai, Y, \& Lavely, W (2003). 'China's Missing Girls: Numerical Estimates and Effects on Population Growth,' The China Review, vol. 3, no. 2, pp. 13-29.

Caldwell, JC (1986), 'Routes to low mortality in poor countries,' Population and Development Review, vol. 12, no.2, pp. 171-220.

Castelli, A \& Nizalova, O (2011). 'Avoidable mortality: what it means and how it is measured' CHE Research Paper \#63, Centre for Health Economics, the University of York.

Centers for Diseases Control and Prevention (CDC) (1986) 'Premature mortality in the United States: Public health issues in the use of potential life lost,' Morbidity and Mortality Weekly Report Supplement 35 (2S), 1s-11s.

Coale, AJ, \& Kisker, E (1986) 'Mortality crossover: Reality or bad data' Population Studies, vol. 40, no. 3, pp.389-401.

Coelli, T, Rao, DSP, O'Donnell, CJ \& Battese, GE (2005). An Introduction to Efficiency and Productivity Analysis, $2^{\text {nd }}$ edn., Springer, New York.

Erreygers, G (2009). 'Correcting the Concentration Index.' Journal of Health Economics, vol. 28 , no. 2 , pp. 504-515.

Farr, W (1885), Methods for comparing local with standard death-rates, Office of the Sanitary Institute, London.

Fleurbaey, M, \& Schokkaert E. (2009). 'Unfair inequalities in health and health care'. Journal of Health Economics, 28, no.1, pp.73-90.

Jonhansson, S, \& Nygren, O (1991). 'The Missing Girls of China: A New Demographic Account', Population and Development Review, vol. 17, no. 1, pp.35-51.

Kjellsson, G., Gerdtham, U.-G. (2013). 'Correcting the Concentration Index for Binary Variables', Journal of Health Economics, vol. 32, no. 3, pp. 659-670. 
Kossarova, L, Holland, W, Nolte, E, \& McKee, M (2009) 'Measuring Avoidable Mortality: Methodological Note', Social and Demographic Analysis, European Commission.

Le Grand, J (1987) 'Inequality in health: some international comparisons', European Economic Review vol. 31, issue 1-2, pp. 182-191.

Le Grand, J (1989) ‘An international comparison of distributions of ages-at-death', in Health Inequalities in European Countries, J. Fox, Editor. Gower: Aldershort. pp. 75-91.

Mackenbach, J \& Looman, C (2013) 'Life expectancy and national income in Europe, 19002008: an update of Preston's analysis' The International Journal of Epidemiology, vol. 42, no. 4, pp. $1100-1110$.

McCracken, K (2002) 'What if New South Wales was more equal?' NSW Public Health Bulletin, vol. 13, no. 6, pp. 123-127.

Nolte, E \& McKee, CM. (2004). Does health care save lives? Avoidable mortality revisited. London, The Nuffield Trust.

Norheim, OF (2010) "Gini Impact Analysis: Measuring Pure Health Inequity before and after Interventions" Public Health Ethics, vol. 3, issue 3, pp. 282-292.

Petrie, D \& Tang, KK (2014), 'Relative health performance in the BRICS over the last 20 years: The winners and losers', Bulletin of the World Health Organization, forthcoming.

Preston, SH (1975), 'The changing relation between mortality and level of economic development,' Population Studies, vol. 29, no.2, pp.231-248.

Preston, S, Elo, IT \& Steward, Q (1999), 'Effects of age misreporting on mortality estimates at older ages' Population Studies, vol. 53, no. 2, pp. 165-177.

Rodriguez, M, and Lopez-Valcarcel, B (2011) "Alas, there are no shortcuts to the complexities of the economy" Journal of Epidemiology \& Community Health vol. 65, issue 5, pp. 389-390.

Romeder, J-M \& McWhinnie, JR (1977). 'Potential years of life lost between ages 1 and 70: An indicator of premature mortality for health planning' International Journal of Epidemiology, vol. 8, no. 2, pp. 143-151.

Rutstein DD, Berenberg W \& Chalmers TC, et al. (1976) 'Measuring the quality of medical care' The New England Journal of Medicine, vol. 294, no.11, pp. 582-88

Salomon J, Tandon, A \& Murray, C. (2004) 'Comparability of self rated health: cross sectional multi-country survey using anchoring vignettes’ $B M J$, vol.328, no.7434: 258

Tang, KK, \& Rao, DSP (2008), 'Measuring Social Disadvantage with Avoidable Mortality Risks" in Clem Tisdell (ed.) Poverty, Poverty Alleviation, and Social Disadvantage, 2008 p.117-131.

Tang, KK, Chin, JTC \& Rao, DSP (2008), 'Avoidable mortality risks and measurement of wellbeing and inequality', Journal of Health Economics, vol. 27, no. 3, pp. 624-641.

Tang, KK, Petrie, D \& Rao, DSP (2007), 'Measuring health inequalities between genders and age groups with Realization of Potential Life Years (RePLY)', Bulletin of the World Health Organization, vol. 85, no. 9, pp. 681-688.

Tang, KK, Petrie, D \& Rao, DSP (2009a). 'Measuring Health Inequality with Realization of Potential Life Years (RePLY)', Health Economics, vol. 18, no. S1, pp. s55-s75.

Tang, KK, Petrie, D \& Rao, DSP (2009b). 'The income-climate trap of health development: A comparative analysis of African and Non-African countries', Social Science \& Medicine, vol. 69, no. 7, pp. 1099-1106. 
Templeton, AR (1998), 'Human races: A genetic and evolutionary perspective', American Anthropologist, vol. 100, no. 3, pp. 632-650.

Uemura, K (1989). 'Excess mortality ratio with reference to the lowest age-sex-specific death rates amongst countries', World Health Statistics Quarterly, vol. 42, pp. 26-41.

Van Doorslaer, E., \& Gerdtham, U. G. (2003). Does inequality in self-assessed health predict inequality in survival by income? Evidence from Swedish data. Social Science \& Medicine, 57(9), 1621-1629.

Wagstaff, A (2005). 'The bounds of the concentration index when the variable of interest is binary, with an application to immunization inequality.' Health Economics, 14: 429432.

Woolsey, TD (1981). Toward an index of preventable mortality, 85, Department of Health and Human Services, Hyattsville.

World Health Organization (WHO) (2012). Life Tables for WHO Member States. Available at: http://www.who.int/whosis/database/life_tables/life_tables.cfm. Accessed 2012.

World Health Organization (WHO) (2013). Handbook on Health Inequality Monitoring: with a special focus on low- and middle-income countries, Geneva, Switzerland.

Zaridze, D., Lewington, S., Boroda, A., Scélo, G., Karpov, R., Lazarev, A., Konobeevskaya, I., Igitov, V., Terechova, T., Boffetta, P., Sherliker, P., Kong, X., Whitlock, G., Boreham, J., Brennan, P., Peto, R. (2014) 'Alcohol and mortality in Russia: prospective observational study of 151000 adults', The Lancet, forthcoming. 


\section{Table 1 Countries with the lowest mortality risks after controlling for income}

\begin{tabular}{|c|c|c|}
\hline Age & Male & Female \\
\hline $0-1$ & Sweden, Slovenia, Estonia, Sri Lanka, Viet Nam, Eritrea, DRC & Singapore, Slovenia, Estonia, Republic of Moldova, Eritrea, DRC \\
\hline $1-5$ & $\begin{array}{l}\text { Finland, Malaysia, FYRM, Republic of Moldova, Kyrgyzstan, Nepal, Eritrea, } \\
\text { DRC }\end{array}$ & $\begin{array}{l}\text { Republic of Korea, Albania, Armenia, Republic of Moldova, Vietnam, Nepal, } \\
\text { Eritrea, DRC }\end{array}$ \\
\hline $5-10$ & Slovenia, FYRM, Tajikistan, Eritrea, DRC & Sweden, Bosnia and Herzegovina, Tajikistan, Eritrea, DRC \\
\hline $10-15$ & Norway, Japan, FYRM, Tajikistan, Eritrea, DRC & Estonia, FYRM, Tajikistan, Eritrea, DRC \\
\hline $15-20$ & Niger, DRC & Israel, Bosnia and Herzegovina, Armenia, Tajikistan, Eritrea, DRC \\
\hline $20-25$ & Netherlands, Niger, DRC & Slovenia, FYRM, Republic of Moldova, Tajikistan, Eritrea, DRC \\
\hline $25-30$ & Kuwait, Netherlands, Morocco, Tajikistan, Niger, DRC & $\begin{array}{l}\text { Singapore, Bosnia and Herzegovina, Armenia, Republic of Moldova, Tajikistan, } \\
\text { Eritrea, DRC }\end{array}$ \\
\hline $30-35$ & Netherlands, Morocco, Tajikistan, Niger, DRC & $\begin{array}{l}\text { Israel, Croatia, Bosnia and Herzegovina, Republic of Moldova, Viet Nam, } \\
\text { Tajikistan, Eritrea, DRC }\end{array}$ \\
\hline $35-40$ & Kuwait, Sweden, Morocco, Tajikistan, Niger, DRC & Singapore, Bosnia and Herzegovina, Tajikistan, Eritrea, DRC \\
\hline $40-45$ & Kuwait, Morocco, Tajikistan, Niger, DRC & Singapore, Greece, Bosnia and Herzegovina, Sri Lanka, Tajikistan, Eritrea, DRC \\
\hline $45-50$ & Kuwait, Sweden, Morocco, Tajikistan, Niger, DRC & Kuwait, Italy, Republic of Korea, Chile, Sri Lanka, Viet Nam, Eritrea, DRC \\
\hline $50-55$ & Kuwait, Italy, Costa Rica, Morocco, Tajikistan, Niger, DRC & Republic of Korea, Sri Lanka, Eritrea, DRC \\
\hline $55-60$ & Kuwait, Peru, Eritrea, DRC & Republic of Korea, Sri Lanka, Eritrea, DRC \\
\hline $60-65$ & Australia, Peru, Tajikistan, Eritrea, DRC & Japan, Republic of Korea, Sri Lanka, Eritrea, DRC \\
\hline $65-70$ & Australia, Ecuador, Nicaragua, Eritrea, DRC & Japan, Spain, Ecuador, Eritrea, DRC \\
\hline $70-75$ & Australia, Ecuador, Nicaragua, Eritrea, DRC & Japan, Ecuador, Nicaragua, Eritrea, DRC \\
\hline $75-80$ & Switzerland, Ecuador, Nicaragua, DRC & Japan, Ecuador, Nicaragua, Eritrea, DRC \\
\hline $80-85$ & Japan, Nicaragua, DRC & Japan, Nicaragua, DRC \\
\hline $85-90$ & Colombia, Nicaragua, DRC & Japan, Nicaragua, DRC \\
\hline $90-95$ & Colombia, Nicaragua, DRC & Nicaragua, DRC \\
\hline $95-100$ & Colombia, Nicaragua, DRC & Nicaragua, DRC \\
\hline
\end{tabular}

Bold frontier countries represent those with the lowest overall mortality rate and the frontier countries are ordered from richest to poorest in terms of their GNI per capita.

FYRM - Former Yugoslav Republic of Macedonia; DRC - Democratic Republic of the Congo 


\section{Table 2 Results for RePLY and RCPLY}

\begin{tabular}{|c|c|c|c|c|c|c|c|c|c|c|c|c|c|c|}
\hline & RePLY & RCPLY & & RePLY & RCPLY & & RePLY & RCPLY & & RePLY & RCPLY & & RePLY & RCPLY \\
\hline Albania & 0.88 & 0.923 & Costa Rica & 0.942 & 0.976 & Iran & 0.871 & 0.902 & New Zealand & 0.962 & 0.969 & Sudan & 0.705 & 0.817 \\
\hline Algeria & 0.866 & 0.906 & Côte d'Ivoire & 0.606 & 0.7 & Ireland & 0.949 & 0.951 & Nicaragua & 0.884 & 0.957 & Swaziland & 0.583 & 0.622 \\
\hline Angola & 0.626 & 0.691 & Croatia & 0.911 & 0.93 & Israel & 0.971 & 0.979 & Niger & 0.689 & 0.87 & Sweden & 0.97 & 0.971 \\
\hline Argentina & 0.901 & 0.931 & Czech Rep. & 0.924 & 0.935 & Italy & 0.976 & 0.979 & Nigeria & 0.644 & 0.745 & Switzerland & 0.979 & 0.98 \\
\hline Armenia & 0.837 & 0.897 & Dem. Rep. Congo & 0.587 & 1 & Japan & 0.989 & 0.99 & Norway & 0.965 & 0.965 & Syrian & 0.882 & 0.942 \\
\hline Australia & 0.976 & 0.978 & Denmark & 0.944 & 0.945 & Jordan & 0.854 & 0.91 & Oman & 0.891 & 0.902 & Tajikistan & 0.81 & 0.939 \\
\hline Austria & 0.957 & 0.959 & Dominican Rep. & 0.856 & 0.901 & Kazakhstan & 0.771 & 0.805 & Pakistan & 0.761 & 0.85 & Thailand & 0.839 & 0.88 \\
\hline Azerbaijan & 0.82 & 0.884 & Ecuador & 0.901 & 0.944 & Kenya & 0.717 & 0.838 & Panama & 0.914 & 0.949 & FYR Macedonia & 0.889 & 0.927 \\
\hline Bangladesh & 0.781 & 0.917 & Egypt & 0.85 & 0.904 & Kuwait & 0.935 & 0.935 & Papua New Guinea & 0.759 & 0.864 & Togo & 0.707 & 0.862 \\
\hline Belarus & 0.838 & 0.873 & El Salvador & 0.858 & 0.904 & Kyrgyzstan & 0.795 & 0.905 & Paraguay & 0.891 & 0.953 & Trinidad and Tobago & 0.842 & 0.854 \\
\hline Belgium & 0.954 & 0.956 & Eritrea & 0.79 & 0.99 & Lao & 0.757 & 0.869 & Peru & 0.904 & 0.949 & Tunisia & 0.899 & 0.939 \\
\hline Benin & 0.684 & 0.798 & Estonia & 0.895 & 0.914 & Latvia & 0.862 & 0.886 & Philippines & 0.835 & 0.905 & Turkey & 0.893 & 0.921 \\
\hline Bolivia & 0.818 & 0.879 & Ethiopia & 0.652 & 0.841 & Lebanon & 0.889 & 0.92 & Poland & 0.904 & 0.926 & Turkmenistan & 0.761 & 0.813 \\
\hline Bosnia and Herzegovina & 0.905 & 0.947 & Finland & 0.953 & 0.955 & Lesotho & 0.576 & 0.671 & Portugal & 0.947 & 0.958 & Uganda & 0.625 & 0.768 \\
\hline Botswana & 0.725 & 0.749 & France & 0.969 & 0.972 & Lithuania & 0.873 & 0.895 & S Korea & 0.955 & 0.963 & Ukraine & 0.811 & 0.857 \\
\hline Brazil & 0.878 & 0.912 & Gabon & 0.741 & 0.764 & Madagascar & 0.775 & 0.943 & Rep. Moldova & 0.828 & 0.91 & United Kingdom & 0.956 & 0.958 \\
\hline Bulgaria & 0.88 & 0.911 & Gambia & 0.716 & 0.822 & Malawi & 0.568 & 0.73 & Romania & 0.879 & 0.911 & Tanzania & 0.666 & 0.801 \\
\hline Burkina Faso & 0.628 & 0.763 & Georgia & 0.847 & 0.911 & Malaysia & 0.88 & 0.906 & Russian Federation & 0.814 & 0.84 & USA & 0.936 & 0.937 \\
\hline Burundi & 0.601 & 0.819 & Germany & 0.957 & 0.959 & Mali & 0.636 & 0.783 & Rwanda & 0.705 & 0.868 & Uruguay & 0.904 & 0.937 \\
\hline Cambodia & 0.733 & 0.856 & Ghana & 0.723 & 0.855 & Mauritania & 0.697 & 0.788 & Saudi Arabia & 0.865 & 0.876 & Uzbekistan & 0.822 & 0.924 \\
\hline Cameroon & 0.612 & 0.693 & Greece & 0.957 & 0.964 & Mauritius & 0.871 & 0.9 & Senegal & 0.74 & 0.846 & Venezuela & 0.895 & 0.926 \\
\hline Canada & 0.967 & 0.969 & Guatemala & 0.829 & 0.885 & Mexico & 0.904 & 0.929 & Sierra Leone & 0.59 & 0.761 & Viet Nam & 0.863 & 0.964 \\
\hline Central African Rep. & 0.578 & 0.735 & Guinea & 0.625 & 0.765 & Mongolia & 0.831 & 0.906 & Singapore & 0.974 & 0.975 & Yemen & 0.78 & 0.875 \\
\hline Chad & 0.574 & 0.695 & Guinea-Bissau & 0.586 & 0.713 & Morocco & 0.87 & 0.934 & Slovakia & 0.898 & 0.917 & Zambia & 0.576 & 0.697 \\
\hline Chile & 0.939 & 0.965 & Honduras & 0.833 & 0.903 & Mozambique & 0.585 & 0.757 & Slovenia & 0.942 & 0.95 & & & \\
\hline China & 0.885 & 0.947 & Hungary & 0.886 & 0.903 & Namibia & 0.687 & 0.728 & South Africa & 0.652 & 0.68 & & & \\
\hline Colombia & 0.912 & 0.951 & India & 0.774 & 0.866 & Nepal & 0.805 & 0.965 & Spain & 0.972 & 0.977 & & & \\
\hline Congo & 0.655 & 0.732 & Indonesia & 0.819 & 0.89 & Netherlands & 0.961 & 0.963 & Sri Lanka & 0.847 & 0.912 & & & \\
\hline
\end{tabular}


Table 3 Correlation of RCPLY-based longevity inequality outcomes using different inequality indexes

$\begin{array}{lccccc}\text { Pearson correlation } & \text { Gini (shortfall) } & \text { Erreygers } & \text { Wagstaff } & \text { Theil } & \text { Atkinson (e=0.5) } \\ \text { Gini } & -0.697 & 0.992 & -0.252 & 0.965 & 0.952 \\ \text { Gini (shortfall) } & & -0.694 & 0.390 & -0.555 & -0.530 \\ \text { Erreygers } & & & -0.231 & 0.955 & 0.947 \\ \text { Wagstaff } & & & -0.188 & -0.178 \\ \text { Theil } & & & & 0.998 \\ & & & & \\ \text { Rank correlation } & \text { Gini (shortfall) } & \text { Erreygers } & \text { Wagestaff } & \text { Theil } & \text { Atkinson (e=0.5) } \\ \text { Gini } & -0.741 & 0.999 & -0.171 & 0.955 & 0.939 \\ \text { Gini (shortfall) } & & -0.733 & 0.340 & -0.589 & -0.562 \\ \text { Erreygers } & & & -0.161 & 0.958 & 0.942 \\ \text { Wagstaff } & & & -0.115 & -0.116 \\ \text { Theil } & & & & & 0.997\end{array}$

Table 4 Pearson correlation coefficients of average longevity and longevity inequality

$\begin{array}{lcccccc} & E(L Y) & E(R e P L Y) & E(R C P L Y) & W(L Y) & W(R e P L Y) & \text { W(RCPLY) } \\ L Y & -0.963 & - & - & -0.629 & - & - \\ \text { RePLY } & - & -0.977 & - & - & 0.467 & - \\ \text { RCPLY } & - & - & -0.973 & - & - & 0.294\end{array}$

Table 5 Average longevity and longevity inequality for China and Russia

$\begin{array}{lccc} & \text { Total } & \text { Males } & \text { Females } \\ \text { China } & & & \\ \text { LY } & 74.0 & 72.2 & 75.8 \\ \text { RePLY } & 0.885 & 0.895 & 0.876 \\ \text { RCPLY } & 0.947 & 0.951 & 0.943 \\ \text { Russia } & & & \\ \text { LY } & & & \\ \text { RePLY } & 68.0 & 61.8 & 74.2 \\ \text { RCPLY } & 0.814 & 0.769 & 0.858 \\ & 0.840 & 0.791 & 0.889\end{array}$


Figure 1 The Preston curve for 1980 and 2010

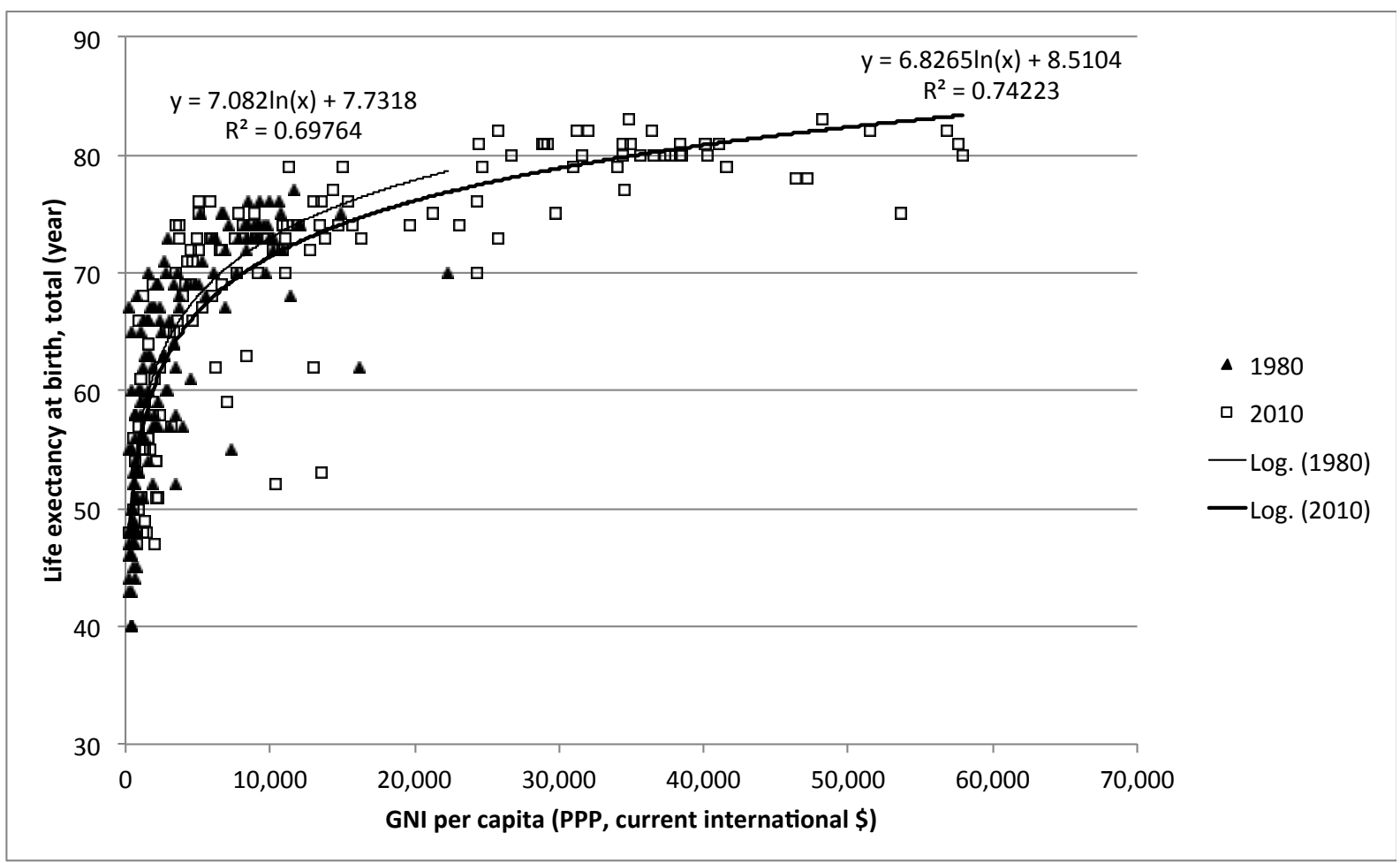


Figure 2 Constructing the global and local frontiers of mortality risk for males aged

\section{between $75-80$}

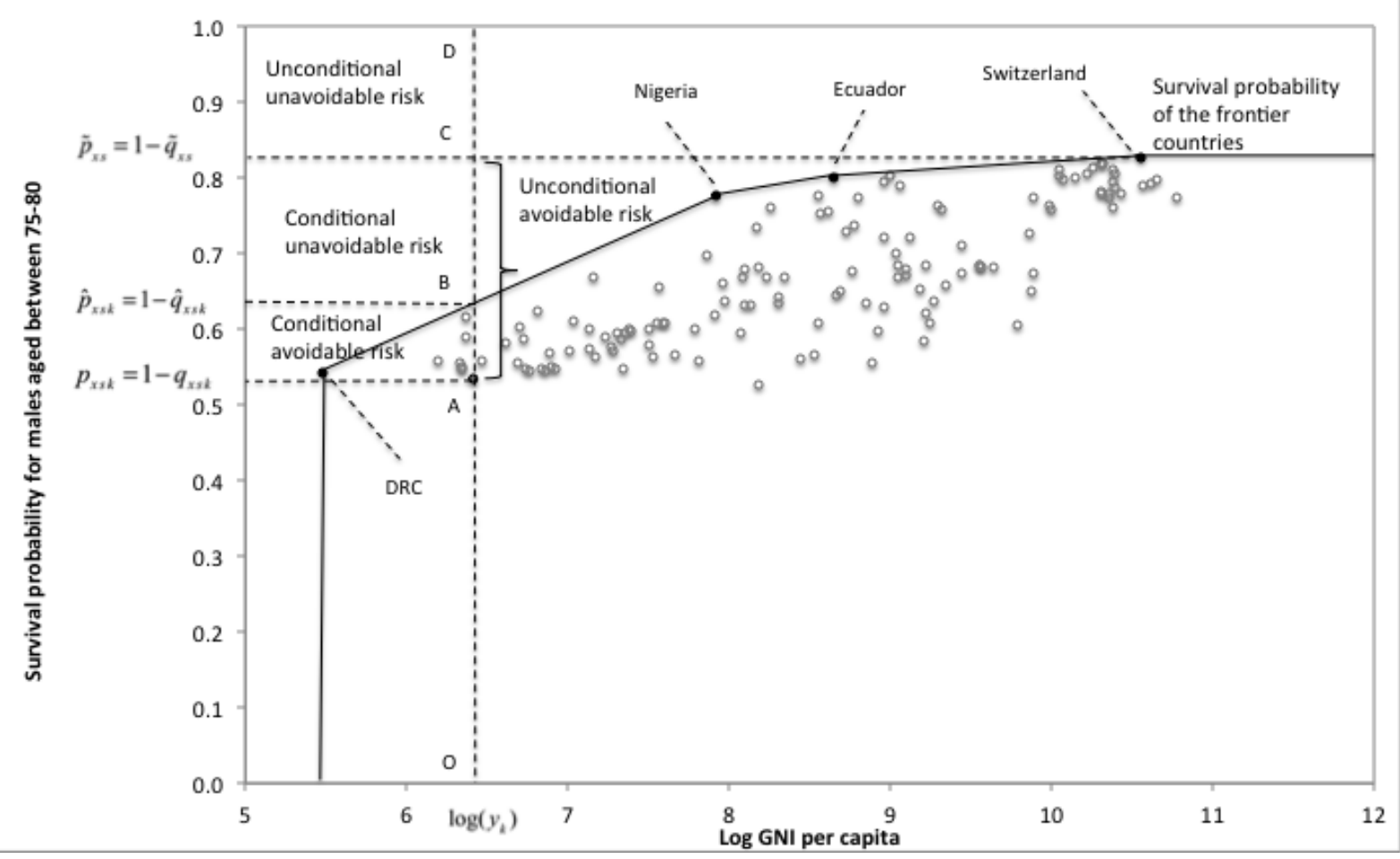


Figure 3 Average RePLY and Average RCPLY for China and Russia
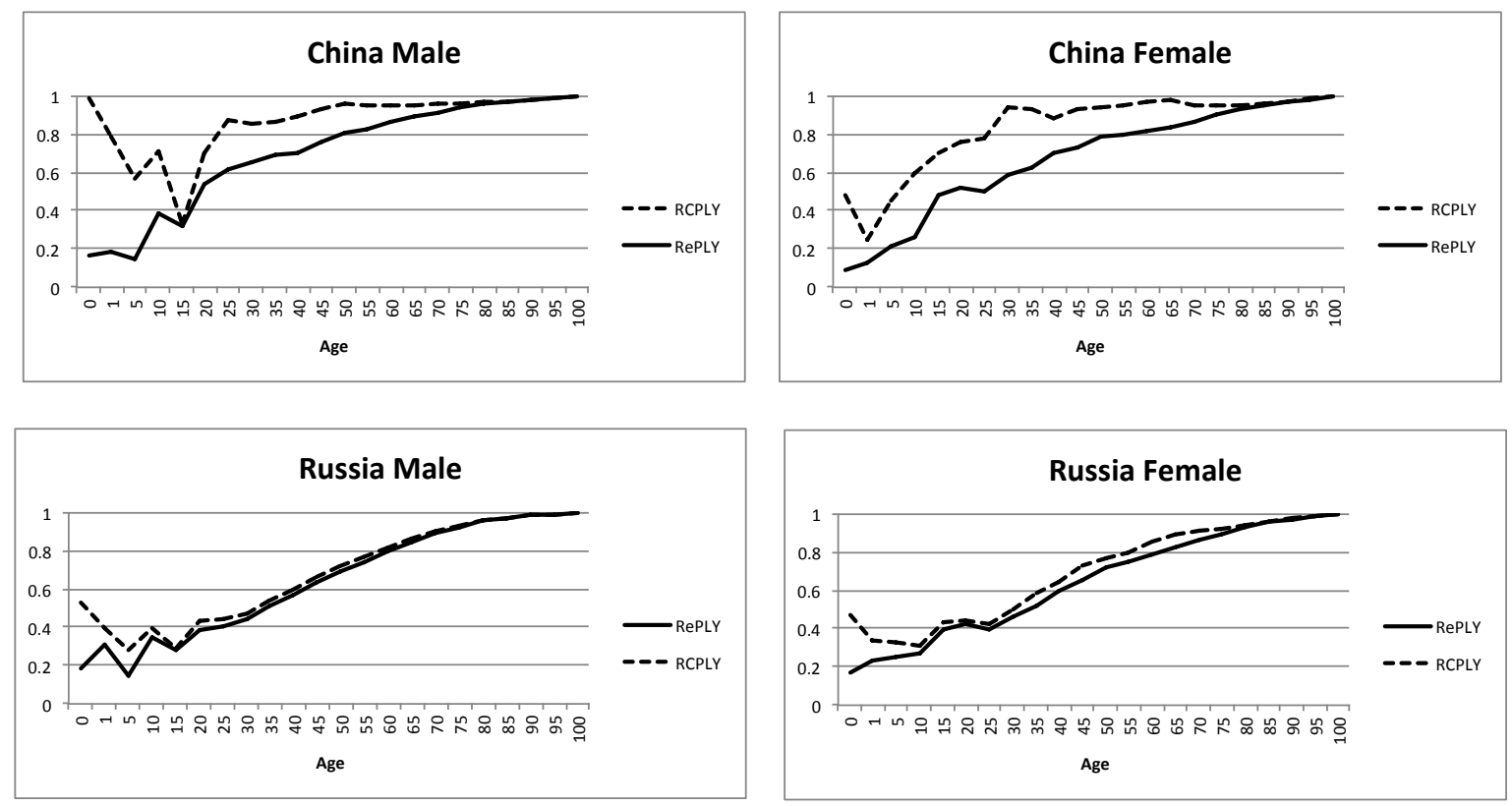
Figure 4 Ratio of Male to Female Average RePLY and Average RCPLY for China

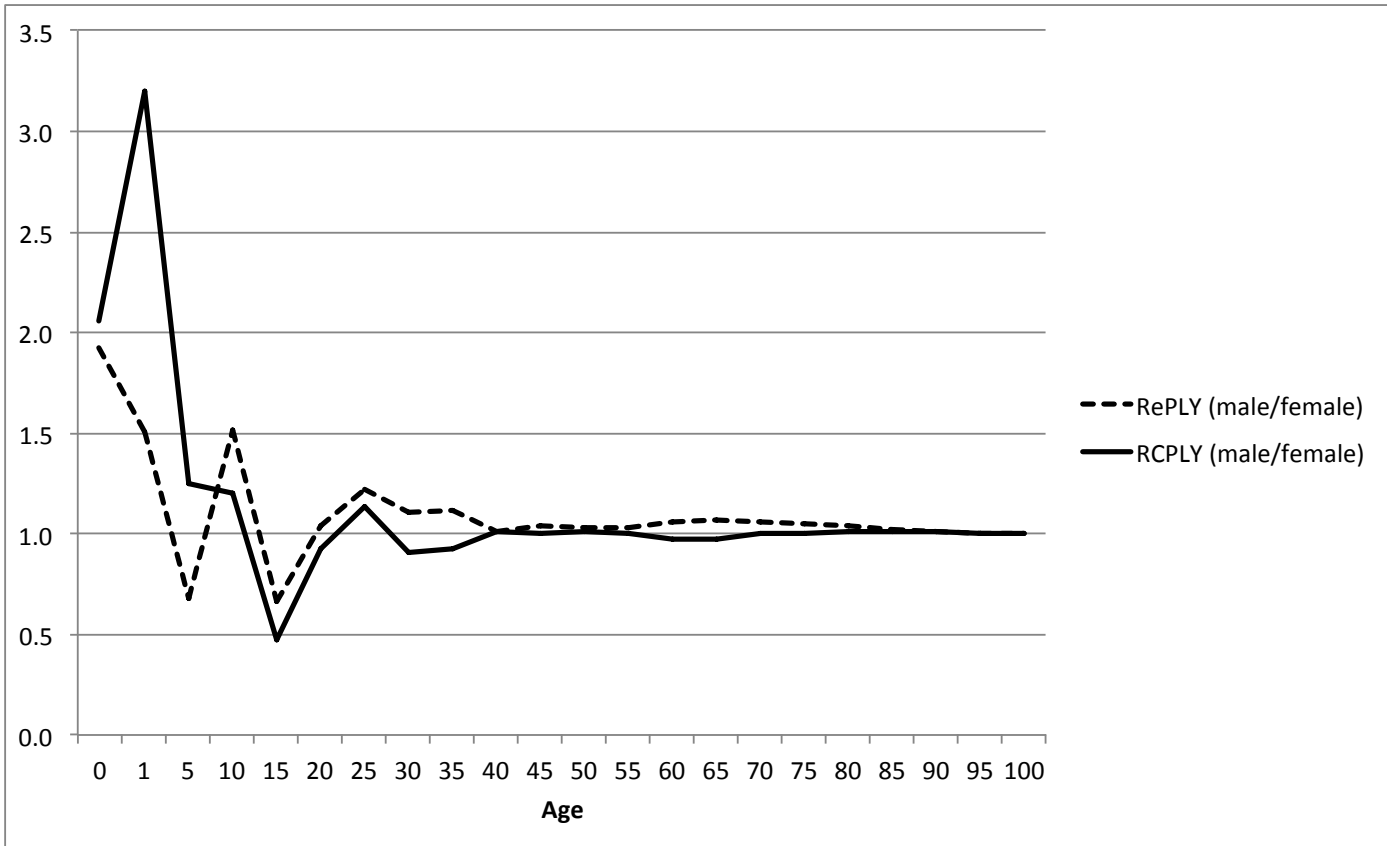


Figure 5 Average RCPLY for China and Russia, 2000 and 2009
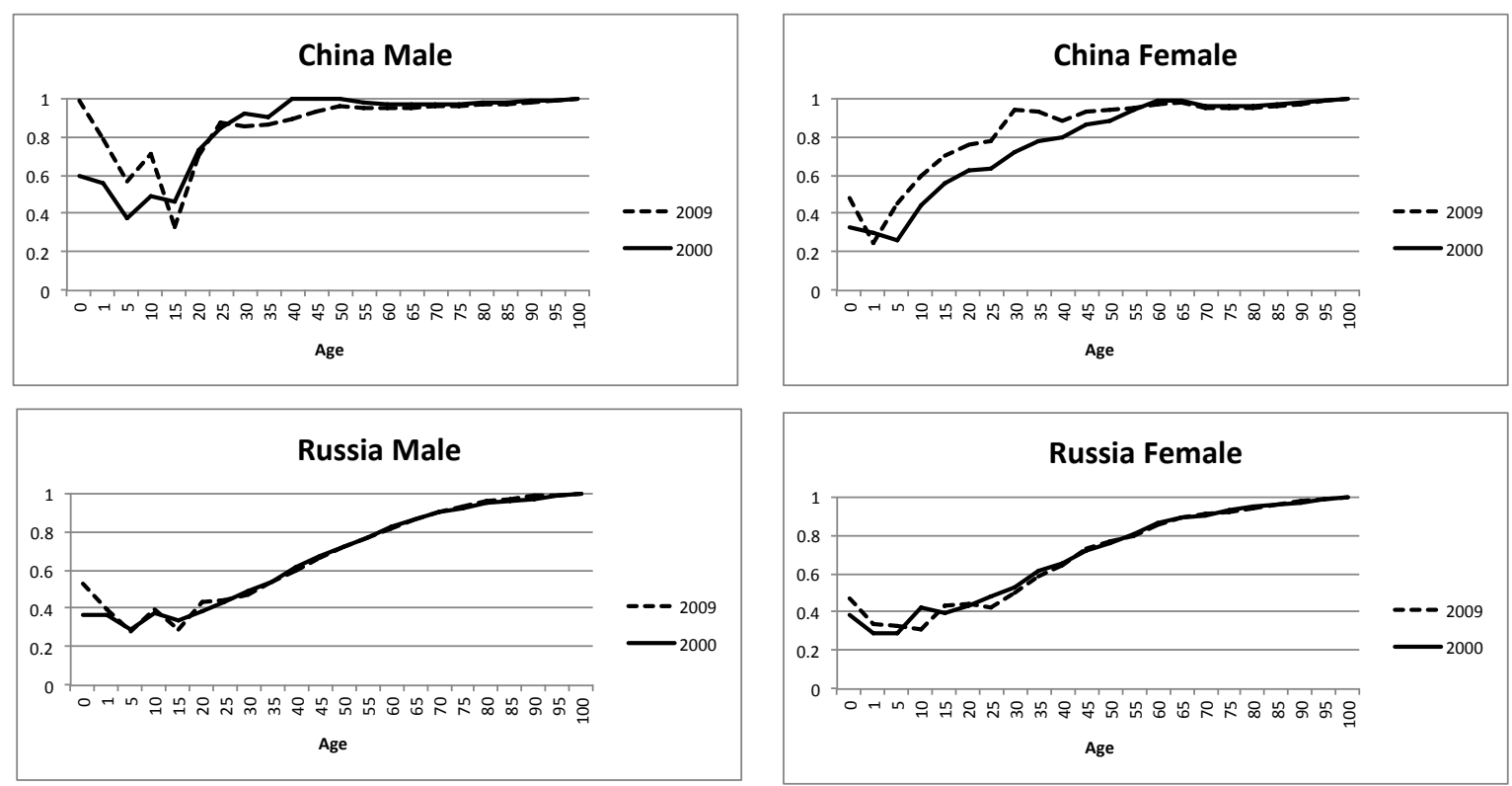